\title{
Caracterización de capas coloreadas sobre AISI 304
}

\author{
J. López ${ }^{(*)}$, E. Blanco $^{(*)}$, J. Botella $^{(*)}$ y L. Esquivias $(* *)$
}

\begin{abstract}
Resumen El método más usual de obtener en la superficie de los aceros inoxidables capas de colores variables es mediante su inmersión en soluciones fuertemente oxidantes $\left(\mathrm{H}_{2} \mathrm{SO}_{4}-\mathrm{CrO}_{3}\right)$ a una temperatura en torno a $70^{\circ} \mathrm{C}$. El trabajo mejora el conocimiento que de estas capas se tiene por medio de técnicas que no eran disponibles hace algunos años, tales como la elipsometría, para la obtención de parámetros ópticos y determinación de espesores, y la microscopía de campo atómico, para la observación de la superficie coloreada. El uso conjunto de estas técnicas permite observar que la película de color está formada por una agregación de partículas con un tamaño que oscila entre los 5 y los $15 \mathrm{~nm}$, los intersticios que aparecen entre las partículas pueden identificarse como poros de unos $5 \mathrm{~nm}$ de tamaño.
\end{abstract}

Palabras clave: Recubrimiento anticorrosivo. AISI 304. Elipsometría. Coloreado.

\section{Characterisation of coloured coatings on AISI 304}

\begin{abstract}
A common method for obtaining colour coatings on stainless steel surfaces is by dipping in strongly oxidizing solutions $\left(\mathrm{H}_{2} \mathrm{SO}_{4}-\mathrm{CrO}_{3}\right)$ at $70{ }^{\circ} \mathrm{C}$. This work improves the knowledge of these coatings by means of techniques that were not available some years ago, such as ellipsometry, to determine the refraction index and the thickness, and atomic field microscopy (AFM), to observe the coloured surface. From these techniques, it is possible to state that films are formed by an aggregation of particle ranging in size from 5 to $15 \mathrm{~nm}$, the interstices are pores of approximately $5 \mathrm{~nm}$ diameter.
\end{abstract}

Keywords: Anticorrosive coating. AISI 304. Ellipsometry. Colouring.

\section{INTRODUCCIÓN}

Desde que existe el acero inoxidable se sabe que sus características de alta resistencia a la oxidación están íntimamente relacionadas con procesos de formación de finas capas de óxidos. Con objeto de conocer el papel exacto que desempeñan las películas de óxidos en los fenómenos de pasividad, Rhodin (1) realizó una clasificación de películas formadas en ácidos oxidantes en caliente en la que señaló la existencia de películas de $40 \mathrm{~nm}$ de espesor que producen colores interferenciales por reflexión de la luz en la superficie metálica. A pesar de conocerse el fenómeno del coloreado desde 1927 (2), sólo tuvo aplicación industrial cuando en la década de los setenta se resolvieron su baja resistencia al des-

(*) ACERINOX. (Los Barrios). 11379 Algeciras. Cádiz (España).

(**) Dpto. de Física de la Materia Condensada. Fac. de Ciencias. Univ. de Cádiz (España). gaste y al rayado. La novedad consistía en el desarrollo de un proceso de endurecimiento mediante un tratamiento catódico en solución ácida (3). Estas capas están compuestas principalmente por hierro, cromo y algo de níquel fuertemente hidratados dispuestos en espinela. Maegama et al. (4) dan un mayor porcentaje de cromo en la superficie del material que va disminuyendo hacia el interior a medida que aumenta la concentración de hierro. Los espesores de las capas oscilan entre 100 y $300 \mathrm{~nm}$ en función del color obtenido.

En cuanto a su proceso de formación, se ha propuesto, entre otras explicaciones, que inicialmente se produce un proceso de oxidación del metal que, al cabo de cierto tiempo, es acompañado por un proceso de disolución de la película, de modo que el progresivo aumento de espesor y el consecuente cambio de color se deben a las variaciones de dicho equilibrio (4).

Una de las propiedades más estudiada del acero coloreado conciernen a la selectividad óptica, que lo hacen como captores solares (5 y 6). Al mismo 
tiempo, el estudio de estas propiedades ha llevado al desarrollo de otros tipos de coloreado (5 y 7), que, en algunos casos, incluyen tratamientos en $\mathrm{H}_{2} \mathrm{SO}_{4}$ con aplicación de un potencial externo (4).

El diferente color que adquieren los aceros está ligado, fundamentalmente, al diferente espesor que adquiere la película crecida electroquímicamente sobre la superficie del acero. Con la intención de estudiar los espesores alcanzados en dichas películas, se ha realizado un estudio elipsométrico del sistema ambiente-película-acero de las diferentes muestras preparadas.

\section{PROCEDIMIENTO EXPERIMENTAL}

\subsection{Preparación de las muestras}

Las muestras estudiadas eran substratos de aceros inoxidables austeníticos tipo AISI-304, de superficie especular. Sobre dichos aceros se han crecido películas coloreadas obtenidas en baño químico con una composición de $250 \mathrm{~g} / \mathrm{L}$ de $\mathrm{CrO}_{3}$ y $490 \mathrm{~g} / \mathrm{L}$ de $\mathrm{H}_{2} \mathrm{SO}_{4}$, a una temperatura de $76{ }^{\circ} \mathrm{C}$. En función del tiempo de immersión en el baño, se obtiene una gama de colores en los aceros, pudiendo seguirse el proceso mediante el registro continuo de las diferencias de potencial existentes respecto a una referencia de platino.

\subsection{Elipsometría de reflexión}

La elipsometría de reflexión permite obtener la relación entre los coeficientes de Fresnel para la reflexión de las componentes de luz polarizada paralela, $r_{p}$, y perpendicular, $r_{s}$, al plano de incidencia:

$$
\rho=\frac{r_{p}}{r_{s}}=\tan \Psi e^{j \Delta}
$$

donde $\Psi$ y $\Delta$ son los ángulos elipsométricos. En un sistema ambiente-película-substrato

$$
\begin{aligned}
& \Psi=\tan ^{-1}\left|\rho\left(n_{0}, n_{1}, n_{2}, d, \phi_{0}, \lambda\right)\right| \\
& \Delta=\arg \left[\rho\left(n_{0}, n_{1}, n_{2}, d, \phi_{0}, \lambda\right)\right]
\end{aligned}
$$

donde $n_{i}$ es el índice de refracción del medio $i, d$ es el espesor de la película, $\phi_{0}$ es el ángulo de incidencia y $\lambda$, la longitud de onda de la radiación empleada. A partir de estos ángulos, se calculan los índices de refracción y el espesor de la película por medio de las subrutinas de Macrackins ( 8 y 9) por cálculo interactivo con el software propio del aparato.
Las medidas se obtuvieron con un elipsómetro automático irradiando un área de $2 \mathrm{~mm}^{2}$ con una luz de 632,8 nm de longitud de onda.

Para determinar los espesores correspondientes a cada película, se ha considerado para el ambiente un valor de $n_{0}=1$, lo que nos permite eliminar dos de las siete variables del sistema. Para determinar el índice de refracción del substrato, se ha utilizado una lámina de acero inoxidable de superficie especular, de las mismas características que las utilizadas como substratos. Así, se han determinado las constantes ópticas de los substratos, obteniéndose un valor de 1,98 para la parte real del índice de refracción y de 3,72 para el coeficiente de extinción.

Para determinar las otras tres variables: espesor, parte real del índice de refracción y coeficiente de extinción de las películas se realizaron medidas a múltiples ángulos de incidencia (MAI). En concreto, se han obtenido valores de $\Psi$ y $\Delta$ para tres ángulos de incidencia.

Estas tres medidas se han realizado sobre el mismo punto de la película, para garantizar que sobre el conjunto de valores de $\Psi$ y $\Delta$ obtenidos solamente actúa como variable el ángulo de incidencia. Dicho ángulo de incidencia se selecciona con una precisión de $\pm 0,1^{\circ}$.

\section{RESULTADOS Y DISCUSIÓN}

Se ha realizado el perfil de espesor correspondiente a la muestra de color rosa fuerte, para la que se le ha registrado un potencial de $17 \mathrm{mV}$. En la figura 1 se representan las curvas de nivel correspondientes a un área de dicha muestra. En ella destaca la gran uniformidad, ya que, aunque aparentemente el perfil es abrupto, no hay más que observar la escala de espesores para comprobar que la diferencia de espesor de un extremo a otro está situada en torno a los $10 \mathrm{~nm}$.

En la figura 2 se representa la variación del espesor, índice de refracción y constante de extinción frente a los potenciales registrados. El error en la determinación del espesor está por debajo del $5 \%$ para todos los casos, excepto para los dos primeros valores. Resulta interesante los valores prácticamente constantes obtenidos para la parte real del índice de refracción, $n$, lo que contrasta con una disminución sistemática de la constante de extinción, $k$. El comportamiento del espesor con el potencial sigue una tendencia creciente, destacando la región comprendida entre 12 y $20 \mathrm{mV}$ donde se observa un mayor rendimiento en el crecimiento con el potencial registrado. Igualmente se observa cómo entre 5 y $15 \mathrm{mV}$ las constantes ópticas de la película permanecen inalteradas. Se corrobora la existencia de una relación directa entre el potencial registrado y 


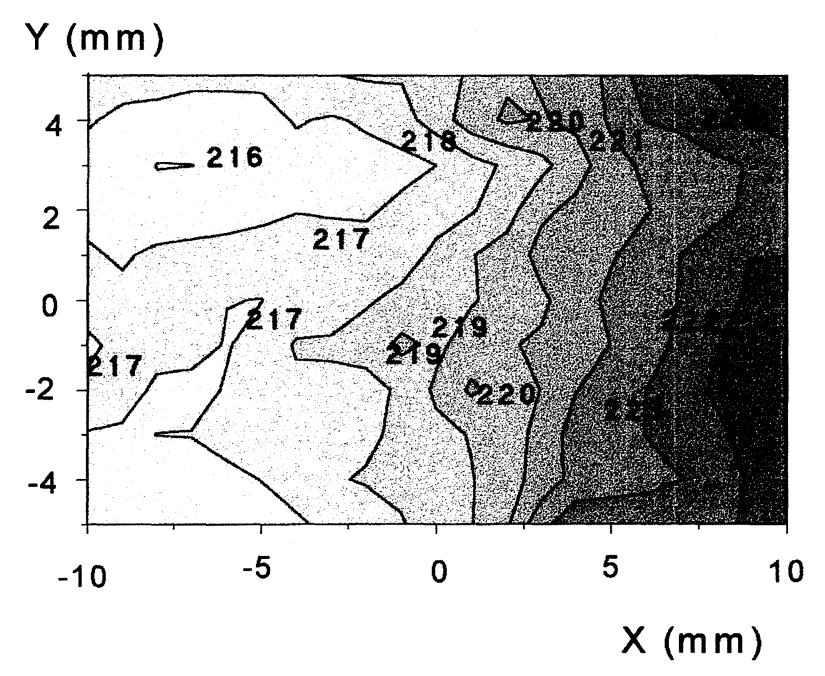

FIG. 1.- Curvas de nivel correspondientes al espesor de la muestra de color rosa fuerte, para la que se ha registrado un potencial de $17 \mathrm{mV}$.

FIG. 1.- Contour lines corresponding to the thickness of the strong pink sample. The potential registered for this sample was $17 \mathrm{mV}$.

el espesor de las láminas obtenido en cada tratamiento.

En la figura 3 se presentan las longitudes de onda a las que corresponde el máximo de interferencia por reflexión para cada potencial, calculándose tanto el primero como el segundo orden de interferencia. La tabla I recoge los espesores correspondientes a cada color, junto con el potencial registrado. Puede observarse que los colores que presentan las muestras con potenciales comprendidos entre 6 y $12 \mathrm{mV}$ se deben al máximo de primer orden. Para las probetas de 15 y $17 \mathrm{mV}$, el color ob-

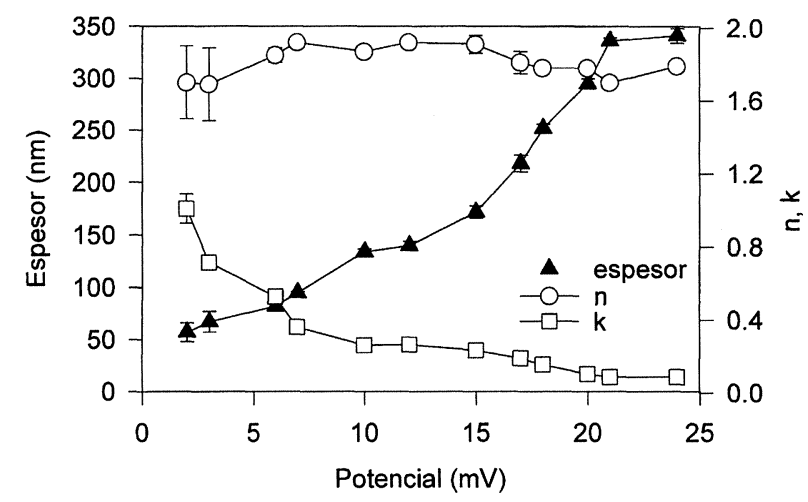

FIG. 2.- Variación del espesor, índice de refracción y constante de extinción frente a los potenciales registrados.

FIG. 2.- Thickness, refraction index and extinction constant vs the registered potentials.

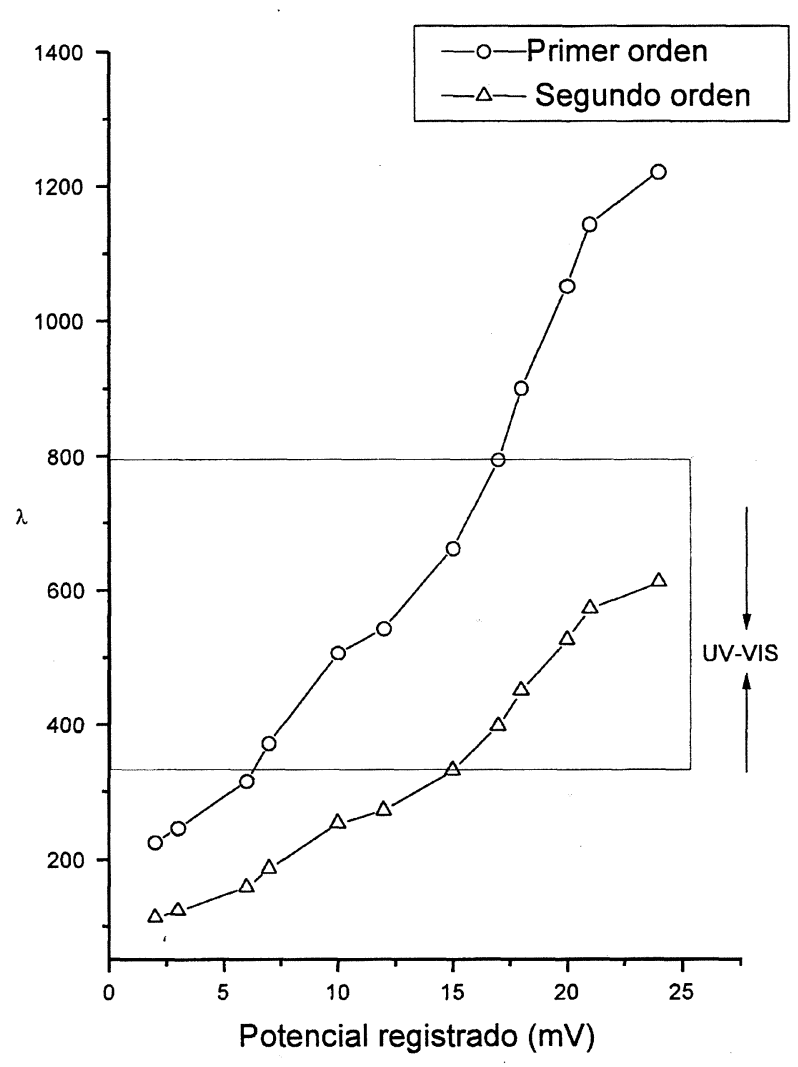

FIG. 3.- Longitudes de onda a las que corresponde el máximo de interferencia por reflexión para cada potencial, calculándose tanto el primero como el segundo orden de interferencia.

FIG. 3.- Wavelength corresponding to the reflection interference maximum for each registered potential. Both first and second order interference maximum were calculated.

tenido es resultado de la mezcla de los correspondientes al primer y segundo orden de interferencia, mientras que para la probeta de $18 \mathrm{mV}$ el color resultante se corresponde con el máximo del segundo orden de interferencia. Para explicar el color de las muestras con potenciales registrados de 2 y $3 \mathrm{mV}$, cuyos máximos se encuentran fuera de la región visible, hay que plantear la condición de mínimo de interferencia, de donde resulta que se extinguen las componentes de 454 y $496 \mathrm{~nm}$, obteniéndose distintas tonalidades de marrón.

La figura 4 muestra la variación de fase en un campo de barridos de $500 \times 500 \mathrm{~nm}$. Estas imágenes muestran que los granos que constituye la capa de color son del orden de los 5-20 nm de diámetro. Al mismo tiempo, permite observar que los intersticios, o poros, existentes entre los distintos granos, se sitúan en torno a los $5 \mathrm{~nm}$, de acuerdo con lo observado por otros autores (10). 
TABLA. I.- Espesores correspondientes a cada color junto con el potencial registrado

TABLE. I.- Thickness corresponding to each obtained colour and the registered potential

\begin{tabular}{|c|c|}
\hline Espesor (nm) & Color/potencial (mV) \\
\hline $57 \pm 9$ & Marrón claroo / 2 \\
\hline $67 \pm 10$ & Marrón / 3 \\
\hline $82 \pm 3$ & Azul marino / 6 \\
\hline $95 \pm 1$ & Azul claro / 7 \\
\hline $134 \pm 3$ & Dorado azulado / 10 \\
\hline $140 \pm 4$ & Dorado / 12 \\
\hline $172 \pm 6$ & Dorado rojizo / 15 \\
\hline $218 \pm 8$ & Rosa fuerte / 17 \\
\hline $252 \pm 4$ & Azul verdoso / 18 \\
\hline $295 \pm 5$ & Verde manzana / 20 \\
\hline $336 \pm 3$ & Verde claro / 21 \\
\hline $341 \pm 7$ & Rosa dorado / 24 \\
\hline
\end{tabular}

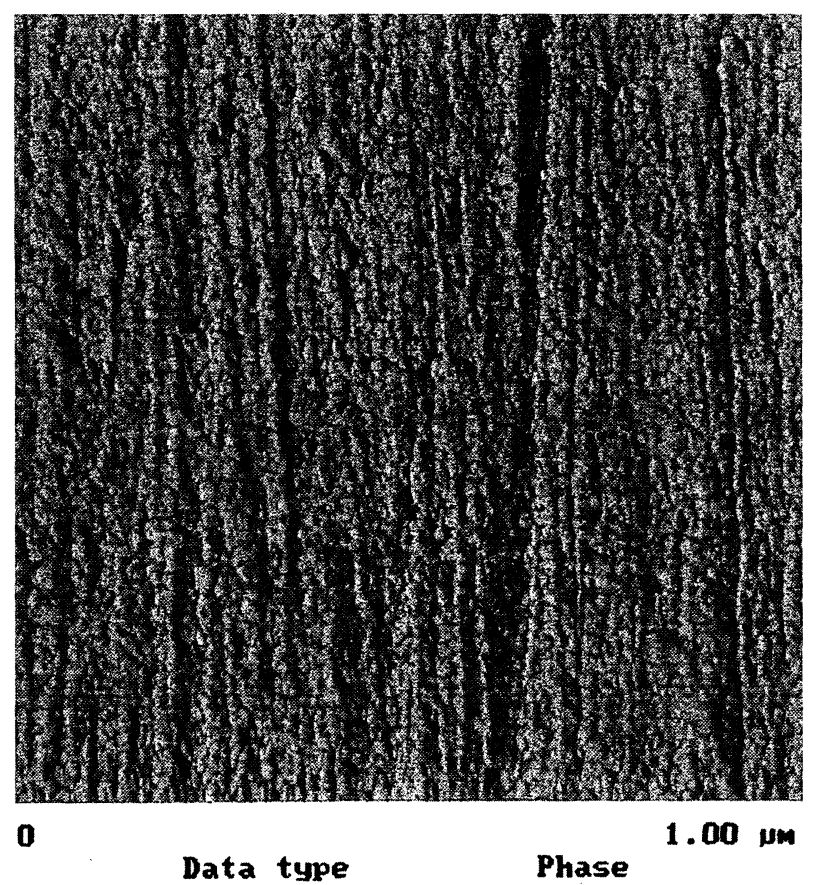

FIG. 4.- Micrografía de correspondiente a la variación de fase AFM de un campo barrido de $500 \times$ $500 \mathrm{~nm}$.

FIG. 4.- Micrograph corresponding to the AFM phase variation of a surface of $500 \times 500 \mathrm{~nm}$.

\section{CONCLUSIÓN}

Se puede confirmar, a partir de estos resultados, la existencia de una relación directa potencial registrado-espesor de la película-color del acero. El color de las muestras con potenciales comprendidos entre 6 y $12 \mathrm{mV}$ se debe al máximo de primer orden. El color de las muestras de 15 y $17 \mathrm{mV}$ es resultado de la mezcla de los correspondientes al primer y segundo orden de interferencia, mientras que, para la probeta de $18 \mathrm{mV}$, su color resultante se corresponde con el máximo del segundo orden. El color térreo de las muestras con potenciales registrados de 2 y $3 \mathrm{mV}$, se debe a que se extinguen las componentes de $454 \mathrm{y}$ $496 \mathrm{~nm}$.

\section{Agradecimiento}

Los autores desean manifestar su agradecimiento a E. Paris, de Instrumat, S.A. por su imprescindible ayuda en la AFM.

\section{REFERENCIAS}

(1) RodHin, T.A.N. Corrosion NACE. 12, 1956: 123-135.

(2) HatFiled, W.H. y Green, H. Patente del R.U. núm. 275 1927: 781 .

(3) Blower, R. y Evans, T.E. Sheet Met. Indust. 51, 1974.

(4) Ohno, J., Abe, S. y Kobayashi, H. Japan J. Appl. Phys. 26, 1987: 1.056.

(5) Balakrishnan, A.R. y Hollands, K.G.T. J. Energy Heat Mass Transfer. 10, 1988: 261.

(6) Venkatachalan, R., Shanmugan, N.V., John, S. y GURUVIAH, S.B. Electrochem. 5, 1998: 182.

(7) Fujimoto, S., Shibata, T., Wada, K. y Tsutae, T. Corr. Sci. 35, 1993: 147.

(8) Macrakins, F.L., Pasaglia, E., Stromberg, R. y SteiBERG, H.L. J. Res. Natl. Bur. Std., 67a, 1963: 363.

(9) Macrakins, F.L. y Colson, J. en Computational techniques for the use of the exact Drude equations in reflection problems. Symp. Ellipsometer and its use in the Measurements of Surfaces and Thin Film, Washintong D.C. 1963. Organizado bajo los auspicios de The National Bureau of Standards, Miscellaneous Publications 256.

(10) Ogura, K., Tsujigo, M., Sakura, K. y Yano, J. $J$. Electrochem. Soc. 140 1993: 1.311. 


\section{CORRIGENDA}

Por indicación del autor Sr. Esquivias, en el artículo "Caracterización de capas coloreadas sobre AISI 304", publicado en Rev. Metal. Madrid, vol. 34, número extraordinario, mayo de 1998, pág 105, debe añadirse al apartado Agradecimiento: "que el Dr. Eduardo Blanco, Profesor de la Universidad de Cádiz, y el resto de autores hacen patente su agradecimiento a la CICYT por el Proyecto MAT94-0676 con el que se financió el trabajo". 